\title{
Use of Machine Learning Methods to Forecast Investment in Russia ${ }^{1}$
}

\author{
Mikhail Gareev, Russian Presidential Academy of National Economy \\ and Public Administration (RANEPA) \\ mkhlgrv@gmail.com
}

This work forecasts the growth rate of quarterly gross fixed capital formation in Russia using machine learning methods (regularisation methods, ensemble methods) over a horizon of up to eight quarters. The methods tested show higher quality in terms of RMSFE than that of simple alternative models (autoregressive model, random walk model), with ensemble methods (boosting and random forest) leading in quality. The last statement is consistent with the results of other research on the application of big data in macroeconomics. It was found that removing observations from the sample which relate to the time before the 1998 crisis and that are atypical for the subsequent period of time does not worsen the short-term forecasts of machine learning methods. Estimates of the coefficients of generally accepted key investment factors obtained using regularisation methods are, on the whole, consistent with economic theory. The forecasts of the author's models are superior in quality to the annual forecasts of growth rates of gross fixed capital formation published by the Ministry of Economic Development.

Keywords: investment

forecasts, machine learning,

LASSO, boosting,

random forest

JEL Codes: C53, E22

\begin{abstract}
Citation: Gareev, M. (2020).
Use of Machine Learning Methods to

Forecast Investment in Russia.

Russian Journal of Money

and Finance, 79(1), pp. 35-56.
\end{abstract}

doi: $10.31477 /$ rjmf.202001.35

\section{Introduction}

Currently, huge arrays of macroeconomic and financial data are available, and many applied studies in economic research come down to retrieving useful information from them. Extensive research has been dedicated to the application of various methods for working with big data volumes in macroeconomics. Models have been developed that make it possible to retrieve information from hundreds of variables and address the challenge of retraining and the so-called 'curse of dimensionality' (see Stock and Watson, 2011). However, the outcomes obtained using many popular machine learning methods are often difficult

\footnotetext{
${ }^{1}$ The author would like to express his gratitude to Andrey Polbin (RANEPA; Caidar Institute for Economic Policy) and anonymous reviewers for their invaluable remarks and corrections.
} 
or impossible to interpret. Researchers must understand how adequate predictive models are and how consistent they are with economic theory, so that the use of regularisation can be justified (i.e. penalising inadequately high coefficients on predictors). Regularisation methods like LASSO (Least Absolute Shrinkage and Selecting Operator) or Ridge, or their modifications, on the one hand, allow the mitigation of risks associated with retraining the models to some extent, and, on the other hand, they preserve interpretability. However, very often methods with weak interpretability tend to demonstrate the highest quality of forecasts in applied research (e.g., ensemble methods like boosting and random forest).

Investments are one of the most important drivers of growth in the long-run, and frequently become the focus of discussions among economists. Research work by Kudrin and Gurvich (2014) focuses on the decrease of investment activity in Russia in 2007-2013, and the restriction of foreign capital inflow and technology imports due to events in Crimea and the South-East of Ukraine. A slowdown in investment activity leads to lagging behind the rest of the world. The low investment attractiveness of the Russian economy, which the authors of the paper believe is primarily caused by weak market mechanisms, is the major obstacle to sustainable growth. Oreshkin (2018) points out that, considering the existing demographic limitations, the only way to boost economic growth rates is to increase the volume and quality of investments. According to estimates of the Ministry of Economic Development (MED), the target GDP growth rate of $3-3.7 \%$ is achievable only through a $25-30 \%$ increase in the share of investments in GDP, and an increase in household savings must be the primary driver of this transformation. In their paper, Idrisov and Sinelnikov-Murylev (2014) pay special attention to institutional drivers for boosting investment prospects in Russia. Among the possible areas of accelerated investment growth, scholars mention advanced technologies (Aganbegyan, 2016) and infrastructure (Oreshkin, 2018).

It is clear that, like any other economic indicator, investments are influenced by many factors, and because it is fundamentally impossible to consider all of them, relatively simple models are often used for forecasting purposes (e.g., an accelerator model implying that the level of investments is defined by the current level of output and its lags). However, it may be possible to extract information from the extensive data of macroeconomic statistics which will help to generate relatively consistent forecasts for investment growth rates. The author hopes that this work, which explores the application of machine learning methods to forecast growth rates for gross fixed capital formation for a horizon of up to 8 quarters, will somehow fill the gap that exists in this area.

There are plenty of examples that demonstrate the successful application of machine learning in macroeconomic forecasts. Thus, Li and Chen (2014) examined the capabilities of LASSO, Elastic Net and Group LASSO in the field of macroeconomic forecasting compared with dynamic factor models (DFMs). 
The data include 107 various monthly indicators for the American economy during the period 1959-2008, and the authors carry out a detailed examination of the results obtained in relation to the 20 most significant indicators. It was found that, in the context of one-step-ahead forecasting, regularisation methods on the whole showed superior quality (in terms of MSFE) as compared with dynamic factor models (DFMs) for 18, 15 and 19 variables out of 20 for LASSO, Elastic Net and Group LASSO. In addition, it was found that combining regularisation methods and DFMs for each of the 20 indicators ensured higher quality forecasts as compared to using DFMs alone.

Among other things, Bai and $\mathrm{Ng}$ (2008) demonstrate that the application of regularisation methods may be useful in terms of determining the factors that impact inflation in the US. On the whole, models that support changes in the set of explanatory variables over time (variables were selected using LASSO, among other things) demonstrated superior quality as compared to models with a fixed set of factors.

In his work, Baybuza (2018) investigates methods to process big data for the purposes of forecasting inflation in Russia. The author uses 92 macroeconomic indicators for the period of 2012-2016 as predictors and generates out-of-sample inflation forecasts for a horizon of 1-24 months. In addition to regularisation methods, ensemble machine learning methods are also considered (random forest and boosting). Models with regularisation yield low-quality forecasts with regard to benchmarks (random walk model, $\mathrm{AR}(1)$ and $\mathrm{AR}(\mathrm{p})$ ) and ensemble methods). However, the combination of $\mathrm{AR}(1)$ and LASSO demonstrates superior quality with regard to forecasts for one month ahead.

A paper by Fokin and Polbin (2019) is dedicated to the alignment of vector autoregression and a LASSO-regularisation (VAR-LASSO) model to forecast the major indicators of the Russian economy: GDP, consumption, and fixed capital investment based on exogenous oil price shock. Based on the testing results, the model offered by the authors demonstrates good predictive power as compared to an ordinary VAR model and to the forecasts of the MED. Furthermore, impulse response functions to the shock in oil prices were constructed.

Hereafter, the work is organised as follows. Section 2 analyses research methodology. First of all, a description is provided for the machine learning methods used (Ridge, LASSO, Post-LASSO, Adaptive LASSO, Elastic Net, Spike and Slab, random forest, boosting) and alternative forecasting methods used for quality comparison. Secondly, the utilised data is described along with the methods of transformation and forecast building. Section 3 contains empirical research results and relevant discussion. The author developed a web application ${ }^{2}$ that enables both the reproduction of the deliverables and the configuration of one's own model specification relative to the boundaries of the training sample and forecasting horizons, as well as the creation of forecasts for investment growth rates. Section 4 presents the conclusion.

\footnotetext{
${ }^{2}$ The application is available at https://mkhlgrv.shinyapps.io/investment_forecasting/
} 


\section{Methodology}

We can highlight several groups of methods for working with the big volumes of data utilised to forecast macroeconomic variables. These differ in terms of their approaches to addressing the 'curse of dimensionality', which is a major obstacle when it comes to using a high number of explanatory variables.

One of the approaches to working with a large number of predictors involves the use of regularisation methods. As we have already mentioned before, the idea is to use a penalty function for increases in the size or number of estimated parameters. The two most common and widely examined regularisers in research papers are LASSO, or regulariser Tibshirani (see Tibshirani, 1996), and Ridge regression, also known as Tikhonov's regulariser, or regulariser (Hoerl and Kennard, 1970).

A great number of poorly interpretable machine learning methods are also available. This research pays special attention to ensemble methods - random forest and boosting.

\subsection{Regularisation method}

Let us assume that a standard linear regression model is defined:

$$
y_{i}=x_{i}^{\prime} \beta+\varepsilon_{i}
$$

where for observation $i=1, \ldots, n, y_{i}$ are the values of the explained variable, $x_{i} \in \mathbb{R}^{p}$ are the values of the $p$ explanatory variables, $\beta \in \mathbb{R}^{p}$ is the vector of $p$ coefficients, $\varepsilon_{i} \sim N\left(0, \sigma^{2}\right)$ are independent and identically distributed random errors. All variables are standardised, i.e. they have zero mean and unit variance. In matrix form, the model is as follows:

$$
Y=X \beta+\varepsilon,
$$

where $Y \in \mathbb{R}^{n}$ are the values of the explained variable, $X \in \mathbb{R}^{n \times p}$ is the matrix of values of explanatory variables, $\beta \in \mathbb{R}^{p}$ is the vector of coefficients, and $\varepsilon \in \mathbb{R}^{n}$ is the vector of independent and identically distributed random errors.

The model is called a sparse linear model with high dimensionality (Belloni and Chernozhukov, 2011a; Belloni and Chernozhukov, 2011b) if it is possible that $p \geq n$, but only $s<n$ elements of $\beta$ are not zero. High dimensionality means that estimation of the model by means of standard OLS may either cause non-robust estimates of the coefficients, or be completely impossible, if $p \geq n$.

If the linear model has high dimensionality, it would be useful to have a method of estimation of coefficients with a smaller variance (even if biased) than when using OLS. However, if the model is a sparse model, then it would be useful to have a way of identifying and discarding the zero elements of vector $\beta$ in addition 
to estimating coefficients. One of the possible ways to address these challenges is to use regularisation methods. A common idea for such methods is to introduce a certain penalty (regularisation operator) that would prevent overfitting caused by unreasonably sizeable estimates of the coefficients, shrinking them toward zero.

\subsubsection{Ridge}

OLS estimations of models with low dimensionality usually have low bias but high variance. However, Ridge regression allows a decrease in variance across estimates of the coefficients, at the expense of making them biased. Formally, the problem looks as follows:

$$
\hat{\beta}^{\text {Ridge }} \in \arg \min _{\beta \in \mathbb{R}^{p}} \lambda\|Y-X \beta\|_{2}^{2}+\lambda\|\beta\|_{2}^{2} .
$$

The standard minimised functional of least squares is compounded with a penalty function that consists of the $l_{2}$-norm of vector $\beta$ multiplied by a penalty parameter value $\lambda$. With $\lambda$ increasing, estimates of the coefficients tend to zero, and $\lambda=0$ is a standard OLS. An important aspect of this approach is the existence of an analytical solution

$$
\hat{\beta}^{\text {Ridge }}=\left(X^{\prime} X+\lambda I_{p}\right)^{-1} X^{\prime} Y .
$$

In case of $\operatorname{rank}(X)<p$, OLS has no solution, because the $X^{\prime} X$ matrix is not invertible. However, a solution exists for the Ridge model, if $\lambda \neq 0$.

The disadvantage of this approach is that it cannot select non-zero coefficients in a sparse model.

\subsubsection{LASSO}

The LASSO method became popular after Tibshirani (1996), however, it had already been mentioned in other publications before that (Santosa and Symes, 1986). A LASSO-based estimate looks as follows:

$$
\hat{\beta}^{\text {LASSO }} \in \arg \min _{\beta \in \mathbb{R}^{p}}\|Y-X \beta\|_{2}^{2}+\lambda\|\beta\|_{1} .
$$

In this case, the sum of the absolute values of the model coefficients multiplied by the parameter $\lambda$ acts as the regularisation operator. As in Ridge, the penalty value is not substantial when $\lambda$ values are low enough, and the results of estimation are similar to those of standard linear regression. However, if the $\lambda$ values are high enough, the model contains no explained variables at all. Unlike Ridge, the use of LASSO allows the selection of just some of the most important variables out of the total set, and the discarding of the rest. However, no analytical solution exists for this model.

Regularisation methods allow the explicit obtainment of estimates of coefficients on the predictors. But can they be interpreted accurately? It is well known that 
coefficients that are estimated using standard LASSO are often unstable over time and can change drastically when new observations are added. This is confirmed, for instance, in Zou and Hastie (2005), and De Mol et al. (2008). There are a number of modifications of classic LASSO that are intended to improve its statistical properties, like Post-LASSO and Adaptive LASSO.

\subsubsection{Post-LASSO}

Normally, estimates by means of regularisation methods will be biased. Belloni and Chernozhukov (2011a) demonstrated that the Post-LASSO method may provide estimates that are potentially less biased than those created by LASSO. The LASSO operator allows the removal of unnecessary variables from the scope of consideration. In this case, it would be quite natural to additionally consider ordinary linear regression after selecting the variables, using only those predictors for which the coefficients were not equal to zero. Thus, we obtain the Post-LASSO estimate. Formally, this may be recorded in the following way:

$$
\hat{\beta}^{\text {Post-LASSO }} \in \arg \min _{\beta \in \mathbb{R}^{p}}\|Y-X \beta\|_{2}^{2} \text {, where } \beta_{j}=0, \quad \text { if } \hat{\beta}_{j}^{\text {LASSO }}=0 \text {. }
$$

Such estimates may provide less bias, however, as Belloni and Chernozhukov (2011a) demonstrated by means of simulation, high data noisiness makes the model unstable, and estimates of the coefficients may be even farther from their true values than those obtained by LASSO.

\subsubsection{Adaptive LASSO}

Zou (2006) demonstrates that in some situations LASSO may incorrectly discard variables (i.e. estimate the coefficients as zero). In some cases, the penalty parameter value of $\lambda$ which shows the highest estimation quality results in the selection of irrelevant variables. However, a situation is also possible in which variables are selected correctly but the non-zero coefficients are unreasonably sizeable, which results in relatively poor forecasts.

Due to this, another LASSO version is offered, Adaptive LASSO, which already uses a weighted penalty function. In this case, subject to certain pre-conditions, the method selects the correct variables, and moreover, we can talk about the consistency of coefficient estimates obtained this way. The improvement suggested by Zou (2006) consists of the preliminary weighting of the vector coefficients $\beta$ which is a part of the regularisation operator $\frac{\lambda}{n}\|\beta\|_{1}$. The problem for finding estimates of the vector of coefficients using adaptive LASSO is formally described as follows:

$$
\hat{\beta}^{\text {Adaptive LASSO }} \in \arg \min _{\beta \in \mathbb{R}^{p}}\|Y-X \beta\|_{2}^{2}+\lambda \sum_{j=1}^{p} w_{j}\left|\beta_{j}\right|,
$$

where $w \in \mathbb{R}^{p}$ is the vector of coefficient weights. According to the results obtained by the authors, if the weights are chosen correctly, the estimates obtained by this 
method are consistent. But how do we choose the weights? The following formula may be used:

$$
w_{j}=\frac{1}{\left(\left|\beta_{j}^{\text {init }}\right|\right)^{\gamma}},
$$

where $\beta_{j}^{\text {init }}$ is the initial estimate of the coefficient $\beta_{j}$ obtained, for instance, using Ridge, $\gamma$ is the additional parameter. With an increase in $\gamma$, the importance of penalty weighting grows (if $\gamma=0$, the problem comes down to ordinary LASSO). Adding weight values allows to specify the variables in the LASSO operator which addition to the model is not desirable (the lower the absolute value of the initial estimate of the coefficient $j$, the higher the penalty for the appearance of the coefficient in the model).

The parameter $\gamma$ could be chosen using cross-validation, though this would not really be reasonable if the available sample is small. According to recommendations provided by the creator of the method, $\gamma=0.5$ is used, and initial weight values are calculated based on Ridge estimates.

\subsubsection{Elastic Net}

Zou and Hastie (2005) demonstrated non-robustness in the selection of variables LASSO caused by parameter uncertainty when estimating the co-variance matrix. To address LASSO this issue, the authors suggested the Elastic Net method. It combines the LASSO and Ridge methods. These methods have the following central features: although LASSO allows the nullification of the coefficients, Ridge yields more robust estimates for highly correlated variables, while LASSO estimates may change substantially when new observations are added. The use of the Elastic Net method allows the nullification of the coefficients (the advantage of LASSO) on the one hand, while the estimates of the coefficients may be relatively robust (the advantage of Ridge) on the other hand. The optimization problem associated with Elastic Net looks as follows:

$$
\hat{\beta}^{\text {Elastic Net }} \in \arg \min _{\beta \in \mathbb{R}^{p}}\|Y-X \beta\|_{2}^{2}+\lambda\left(\alpha\|\beta\|_{1}+(1-\alpha)\|\beta\|_{2}^{2}\right) \text {. }
$$

The regularisation operator consists of the weighted sum of the LASSO and Ridge operators. This definition of the task implies two parameters already: $\lambda$, like the one mentioned above, is responsible for the importance of the penalties, and $\alpha$ weighs Lasso and Ridge against each other. With $\alpha=1$, the model comes down to LASSO, and with $\alpha=0$, to Ridge. The $\alpha$ parameter may be selected by means of cross-validation, however, this is not really justified when the sample is small, so in this research work its value is set at a level of 0.5 , equidistant from the two extremes. The same value was selected, for instance, in Baybuza (2018).

\subsubsection{Spike and Slab}

In econometrics, the Bayesian approach assumes the existence of a prior distribution for each model parameter, the combination of which with the available 
data yields a posterior distribution. This approach, for instance, allows us to obtain estimates for parameters even if the number of variables exceeds the number of observations or to directly pose a question about the likelihood of any of the model's coefficients equal to zero. This research uses the Bayesian Spike and Slab method.

It is important to note that regularisation methods may also be described through the Bayesian approach. In case of normal prior distribution, the Bayesian model comes down to Ridge regression, and when Laplace distribution is used, to LASSO regression (see De Mol et al., 2008).

In a generic form, the representation of the model looks as follows. Let us assume equation (2) holds and the following prior beliefs about parameters of the model are assumed:

$$
\left\{\begin{array}{l}
\left(y_{i} \mid x_{i}, \beta, \sigma^{2}\right) \sim N\left(x_{i}^{\prime} \beta, \sigma^{2}\right) \\
(\beta \mid \gamma) \sim N(0, \Gamma) \\
\gamma \sim \pi(\cdot) \\
\sigma^{2} \sim \mu(\cdot)
\end{array}\right.
$$

where $\sigma^{2}>0$ is the variance of the error term, $\Gamma=\gamma \cdot I_{k}, I_{k}$ is an identity matrix, $\pi(\cdot)$ and $\mu(\cdot)$ are prior distributions of respective parameters.

Various versions of prior distributions exist. The author uses the Spike and Slab method as described in Ishwaran and Rao (2005), which makes the following assumptions:

$$
\left\{\begin{array}{l}
\left(\beta_{j} \mid \tau_{j}, \rho_{j}^{2}\right) \sim N\left(0, \tau_{j} \cdot \rho_{j}^{2}\right), \\
\tau_{j} \sim(1-w) \delta_{v 0}(\cdot)+w \delta_{1}(\cdot), \\
\left(\rho^{-2} \mid \alpha_{1}, \alpha_{2}\right) \sim \Gamma\left(\alpha_{1}, \alpha_{2}\right), \\
w \sim U[0 ; 1],
\end{array}\right.
$$

where $\delta_{x}$ is the discrete measure concentrated in the vicinity of point $x, v_{0}$ is a number close to zero. The number $v_{0}$ and the parameters of Gamma distribution $\alpha_{1}$ and $\alpha_{2}$ are selected in such a way that variance of the coefficient $\gamma_{j}$ has a peak at zero and a right-side tail area. Unlike other earlier versions of the model, this specification assumes the continuity of all distributions used instead of defining them as piecewise functions, which ensures greater flexibility of the model. In accordance with these assumptions, minimisation of the posterior sample mean of errors is performed, which is standard for the Bayesian approach.

\subsection{Ensemble methods}

\subsubsection{Random forest}

Random forest falls under the category of ensemble methods. The general idea behind ensemble methods is the use of a large number of 'simple' regression methods within a single sample or the classification and generation of predicted 
values based on the averaged predictions of these methods. The random forest method described by Liaw and Wiener (2002) is based on building decision trees. What is the motivation for their use? Linear methods of model estimation feature a number of advantages: they can be quickly trained, they support the processing of a large number of conditions, and they can be regularised. However, they still have a serious disadvantage: they are only able to estimate linear dependencies between variables (of course, the model specification may be changed and nonlinear components added, but these changes must be substantiated, and these opportunities are quite limited anyway). Decision trees allow this challenge to be addressed to some extent. Though initially they were used for completing classification tasks (for instance, the classical task solved using trees - the binary classification of potential debtors by a bank, whether or not the debtor will repay the credit), they may also be used for regression tasks. However, the trees themselves are currently seldom used, but often combined into a composition.

Before we proceed with a description of the random forest method, we will give a formal definition of a binary decision tree. Let us assume that an explained variable vector, $Y$, and a matrix of explanatory variables, $X$, are specified. The tree consists of internal and terminal (leaf) nodes. Each internal node $v$ has a $\beta_{v}: X \rightarrow\{0,1\}$ function (predicate) assigned to it, and each terminal node $u$ has a $y_{u} \in Y$ forecast assigned to it. Moreover, algorithm $(X)$ is defined, which starts from an initial node $v_{0}$ and transitions to the left-hand node if $\beta_{v_{0}}(X)=0$, or to the right-hand node if $\beta_{v_{0}}(X)=1$. This happens until the algorithm reaches a terminal node, after which the explained variable is forecast. Usually, one predicate uses just one variable from the set of explanatory variables.

How are the trees constructed? In the construction of each node, the variable and its divisional value are selected in such a way as to ensure the maximum improvement of the pre-defined quality functional. For instance, the sum of errors squared RSS $=\sum_{i=1}^{n}\left(y_{i}-\hat{y}_{i}\right)^{2}$ may act as such a functional, and the mean value or median of the explained variable in the sub-sample may be used as predicted value $\hat{y}_{l}$. The stopping criterion is checked at each node. If the check fails, the node is declared an internal node, and splitting continues. If the check is successful, the node is recognised as a terminal node and is assigned forecast $\hat{y}_{i}$. The maximum tree depth, the minimum number of objects at a node, the maximum growth of the quality functional, or a combination of the above, may be used as a stopping criterion.

It is easy to notice that trees are prone to overfitting (for instance, in a trivial case, you can develop an error-free tree on a training sample if each terminal node contains only one object). Random forest is not so susceptible to this issue. 'Planting' decision trees into a random forest consists of two steps:

1. Data are randomly divided into $N$ sub-samples with replacements. The size of each sub-sample is equal to the size of the entire training sample, but a certain part of the observations is excluded from the sample, and other observations are found multiple times. A decision tree is built on each 
sub-sample. However, when building the tree on a single sub-sample, only $p_{0}$ variables that are randomly selected from $p$ predictor variables individually for each sub-sample are available.

2. The averaged value of $\hat{y}_{i}$ across all $j(j=1, \ldots, N)$ trees is selected as the predicted value $\hat{y}_{i j}$.

This approach allows the achievement of high predictive power, and, as we have already mentioned above, using multiple trees secures the model against overfitting to some extent. However, meaningful economic interpretation of the results of random forest building is quite difficult or completely impossible.

Moreover, the use of decision trees involves an important restriction: predicted values cannot go beyond the values that were observed in the training sample, while such a problem does not exist for linear models. This restriction may be especially important in terms of forecasting macroeconomic or financial data.

\subsubsection{Boosting}

Like random forest, gradient boosting, as proposed in the research by Friedman (2001), belongs to the category of ensemble methods (i.e. it is a composition of several models of the same type). However, unlike random forest, boosting allows the iterative improvement of the model using information obtained from previous learning iteration.

Boosting is actually a general methodology of developing methods, so it can use almost any type of models as base models. However, as with random forest, the base model is often a decision tree. This is the approach that is used in this paper. In the course of boosting, the base model $M_{1}$ is first trained on a training sample, then the model errors are counted, and a new model $m_{2}$ is trained based on them. The new model is added to the previous one with the multiplier $\eta \in(0,1)$ :

$$
M_{2}=M_{1}+\eta m_{2}
$$

The parameter $\eta$ is responsible for the speed of learning (if the speed too high, the model is prone to overfitting, and if too low, then more iterations will be needed to get high-quality predictions).

The result of $N$ iterations is the model $M_{N}=M_{1}+\sum_{i=2}^{N} \eta^{i-1} m_{i^{*}}$

\subsection{Alternative models}

This subsection describes alternative models used in the paper which are not related to machine learning methods.

\subsubsection{Random walk}

A simple random walk model is used as a basic model. Random walk is a simple and clear quality indicator that is frequently used in applied economic 
research. This model assumes that process increments over time are white noise. Formally, a forecast of $h$ steps ahead may be recorded in the following way:

$$
\hat{y}_{t+h}=y_{t}
$$

\subsubsection{Autoregression}

Another frequently used model to compare quality when forecasting time series is the autoregressive model in the range of $p, \operatorname{AR}(p)$. This model assumes that the process changes at a given moment in time $t$ are fully explained by its $p$ lagged values and random error $\varepsilon_{t}$ :

$$
y_{t}=a_{0}+a_{1} y_{t-1}+a_{2} y_{t-2}+\ldots a_{p} y_{t-p}+\varepsilon_{t} .
$$

In practice, the number of lags is usually selected using information criteria, AIC or BIC.

\subsection{Data}

Growth rate values for gross fixed capital formation are used as a predicted variable. This variable consists of three series of gross fixed capital formation provided by Rosstat for different periods (from 1995Q1 to 2002Q4, from 2003Q1 to 2010Q4, and from 2011Q1 to 2019Q1) by means of calculating the 1 st log difference relative to the corresponding quarter of the previous year. This approach allows the removal of seasonality in the series of gross fixed capital formation. Moreover, it accounts for possible multiplicativity and structural shifts in seasonality. The extended Dickey-Fuller test performed reveals no nonstationarity in the transformed series. After its transformation, each time series is reduced by 4 first observations and starts from 1996Q1.

A set of 36 (37, together with the values in predicted series) macroeconomic indicators of the Russian economy were used as predictors.

Unfortunately, many of the indicators frequently used in macroeconomic forecasting appeared in Russia relatively late, in the mid-2000s. This includes the well-known PMI survey indices, which reflect the sentiment of management across various industries. If they were included in the data, then there would be very few quarterly observation models available for learning and quality check, so the author had to sacrifice the number of predictors in order to extend the sample: the source values of all series used in the research are available starting from at least 1995Q1. They were all normalised to stationary form as may be required. This was made by means of calculating the first log difference either relative to the previous quarter (for series without seasonality), or relative to the corresponding quarter of the previous year (for series with seasonality). After all transformations, 1996Q1 was set as the earliest point for the data used 
in the research. A full list of the variables, their sources and their methods of transformation are given in Table 6 in Appendix. Figure 1 in Appendix shows a graph of the standardised ${ }^{3}$ variables, in stationary form, used in the research, along with a separate series of predicted variable.

\subsection{Generating forecasts}

In this research, several specifications were examined for each model.

We analysed two start dates (the left-hand boundaries of the training sample): 1996Q1 and 2000Q1. The first date was chosen only because many macroeconomic indicators were unavailable before 1995, and after the transformations performed with the data, the minimum available date shifts to 1996Q1. The second date was chosen for the following reasons: we can presume that adding information for the period that preceded the 1998 crisis (for 2000Q1, the earliest data used for learning refers to 1999Q1) does not improve the quality of the models. If this hypothesis receives indirect proof, this may also be useful for futher research.

For each specification, the regularisation models were trained in the area between the left-hand and right-hand boundaries of the training sample, where the right-hand boundary takes values in the range from 2012Q1 to 2018Q4, separately for forecasting on horizons of $h$ of 1-8 quarters.

In this case, for models with $h=1$, the end date for the training sample is 2018Q3; for models providing forecasts for 2 quarters, this is 2018Q2, etc.; for models providing forecasts for 8 quarters, this is 2016Q4. Therefore, the range of the training sample extended from 65 to $84(h=8)$ and $91(h=1)$ observations for the first start date (1996Q1), and from 49 to $68(h=8)$ and $75(h=1)$ observations for the second start date (2000Q1).

After training was complete, out-of-sample forecasts were generated for each training sample on the horizon of $h=1,2 \ldots, 8$ quarters for the first observation following the training sample, and for the new iteration, the boundary of the training sample shifted one observation ahead. Therefore, to obtain the forecast for quarter $t+h$, only the values of the variables in quarter $t$ were used.

Parameters of the penalty in corresponding regularisation models were selected using cross-validation in the training sample for each individual specification. Cross-validation inside the training sample was performed in a fixed sliding window of 40 quarters with increments of 1 .

The methodology of forecast generation for random forest and boosting models is not much different from that of forecast generation for regularisation models, except for the fact that cross-validation was not used for selecting parameters.

\footnotetext{
${ }^{3}$ In this figure, standardisation is only required for convenience of graphic representation. In the course of model estimation, standardisation of data was only performed for regularisation methods. It is not required for ensemble methods.
} 
Random forest parameters were selected as follows. The number of variables available for the development of a single decision tree, $p_{0}$, is established at the level recommended by Liaw and Wiener (2002) for $p_{0}=p / 3$ regression tasks, where $p$ is the number of variables. Thus, $p_{0}=12$. When selecting the number of trees $N$, the following guidelines must be observed. With a small number of trees, we cannot be sure that all observations are used in the model; however, as the number of trees increases, the computational complexity also grows. Four different values for $N$ are used in this research work: 100, 500, 1000, 2000. As you will see below, the quality of the results for these four models is similar enough, and none of them leads with regard to all the specifications. However, the difference between $N=1000$ and $N=2000$ is almost imperceptible, so a further increase of $N$ is useless. This research uses the standard stopping criterion for tree development, which is recommended by the authors of the method. A node is recognised as a terminal node if further splitting results in one of the child nodes containing less than 5 observations.

Let us proceed with the boosting parameters. The standard value for learning speed is set at $\eta=0.3$. Though this value usually results in quite a conservative model, additionally test $\eta=0.4, \eta=0.2$ and $\eta=0.1$ specifications. It is not possible to unambiguously select the best parameter value in various specifications. The parameter $N$ is responsible for the number of boosting iterations. Usually, when $N$ is selected, one stops at a level after which the model predictions no longer change. For the purposes of this work, setting $N=100$ was sufficient.

The development of benchmark models is worth a special mention. Forecasts made by the random walk ( $h$ steps ahead) model were merely the current values of the predicted series.

To build the autoregression model, we first selected the number of lags used for each specification (usually no greater than 2) by means of AIC. After this, we estimated the coefficients and generated a recursive forecast 8 steps ahead, i.e. we performed sequential estimation of all 8 predicted values instead of estimating 8 individual equations. As we can see in Faust and Wright (2013), this approach ensures more precise forecasts.

The quality metric used in the research is RMSFE (Root Mean Squared Forecast Error):

$$
\mathrm{RMSFE}=\sqrt{\frac{\sum_{t=1}^{T}\left(\hat{y}_{t}-y_{t}\right)^{2}}{T}} .
$$

This metric can often be seen when solving regression tasks (it is used, for instance, in Fokin and Polbin, 2019; Baybuza, 2018). All calculations ${ }^{4}$ were performed in $\mathrm{R}$ using the following packages:

- glmnet for Ridge, LASSO, Post-LASSO, Adaptive LASSO, Elastic Net,

- spikeslab for Spike and Slab,

\footnotetext{
${ }^{4}$ The code for calculation is available at https://github.com/mkhlgrv/investment_forecasting/
} 
- randomForest for random forest,

- forecast for the AR model,

- xgboost for the boosting model.

\section{Results and discussion}

\subsection{Quality of models}

After generating the forecasts, RMSFE values were calculated for each model in the training sample. These values relative to the quality indicator for the naive forecast (random walk process) for forecasting horizons of 0-8 quarters are listed in Table 1 for the left-hand boundary of the training sample at 1996Q1, and in Table 2 for the right-hand boundary of the training sample at 2000Q1.

Table 1. RMSFE (1996Q1 is used as the left-hand boundary of the training sample)

\begin{tabular}{lcccccccc} 
Model & $\boldsymbol{h}=\mathbf{1}$ & $\boldsymbol{h}=\mathbf{2}$ & $\boldsymbol{h}=\mathbf{3}$ & $\boldsymbol{h = 4}$ & $\boldsymbol{h = 5}$ & $\boldsymbol{h = 6}$ & $\boldsymbol{h}=\mathbf{7}$ & $\boldsymbol{h}=\mathbf{8}$ \\
\hline Random walk & 1.00 & 1.00 & 1.00 & 1.00 & 1.00 & 1.00 & 1.00 & 1.00 \\
\hline AR & 0.92 & 0.85 & 0.79 & 0.73 & 0.68 & 0.67 & 0.68 & 0.61 \\
\hline Adaptive LASSO & 0.99 & 0.91 & 0.79 & 0.84 & 0.75 & 0.77 & 0.61 & 0.50 \\
\hline Elastic Net & 1.00 & 0.85 & 0.84 & 0.80 & 0.76 & 0.74 & 0.69 & 0.56 \\
\hline LASSO & 1.01 & 0.89 & 0.83 & 0.81 & 0.82 & 0.72 & 0.71 & 0.55 \\
\hline Post-LASSO & 1.04 & 0.95 & 0.80 & 0.93 & 0.79 & 0.78 & 0.72 & $\mathbf{0 . 5 4}$ \\
\hline Ridge & 0.98 & 0.88 & 0.88 & 0.87 & 0.81 & 0.79 & 0.70 & 0.61 \\
\hline Spike and Slab & 1.00 & 0.84 & 0.82 & 0.79 & 0.77 & 0.74 & 0.63 & 0.58 \\
\hline Boosting $(\eta=0.1)$ & 0.91 & 0.74 & 0.81 & 0.66 & 0.67 & 0.53 & $\mathbf{0 . 4 5}$ & 0.62 \\
\hline Boosting $(\eta=0.2)$ & $\mathbf{0 . 8 7}$ & 0.76 & 0.79 & 0.71 & 0.68 & $\mathbf{0 . 4 6}$ & 0.49 & 0.59 \\
\hline Boosting $(\eta=0.3)$ & 0.96 & 0.79 & $\mathbf{0 . 6 9}$ & 0.71 & 0.73 & 0.52 & 0.53 & 0.60 \\
\hline Boosting $(\eta=0.4)$ & 1.01 & $\mathbf{0 . 7 0}$ & 0.82 & 0.69 & $\mathbf{0 . 6 1}$ & 0.54 & 0.48 & 0.59 \\
\hline Random forest $(N=100)$ & 0.90 & 0.72 & 0.75 & $\mathbf{0 . 6 5}$ & 0.70 & 0.56 & 0.54 & 0.57 \\
\hline Random forest $(N=500)$ & 0.88 & $\mathbf{0 . 7 0}$ & 0.75 & 0.66 & 0.66 & 0.58 & 0.53 & 0.56 \\
\hline Random forest $(N=1000)$ & 0.90 & $\mathbf{0 . 7 0}$ & 0.76 & 0.66 & 0.66 & 0.58 & 0.55 & 0.55 \\
\hline Random forest $(N=2000)$ & 0.90 & 0.70 & 0.75 & 0.66 & 0.67 & 0.58 & 0.54 & 0.56 \\
\hline
\end{tabular}

Random forest and boosting models almost always produce the best results for the two different start dates in the training sample. This was to be expected; poorly interpretable machine learning methods often demonstrate better results in terms of forecast quality in comparsion with regularisation methods. For instance, this pattern was seen in Baybuza (2018), where random forest and boosting methods turned out to be the winners for almost all specifications in the course of inflation forecasting, or in Kvisgaard (2018), where regularisation methods performed worse than other machine learning methods in the context of GDP and inflation forecasting. Selecting different values for the $N$ parameter produces an insignificant deviation in the forecasting quality of the random forest model, which allows us to assert that a further increase in the number of decision trees will not improve the quality of predictions. 
It is worth noting that the use of LASSO modifications is not justified. Adaptive LASSO and Post-LASSO do not offer better forecasting quality as compared to the 'parent' model. The Bayesian approach to regularisation in the context of this research did not provide any significant improvements to the forecasting quality. Interestingly, the Elastic Net method, which in theory uses the advantages of both Ridge and LASSO, is quite often worse than at least one of these models. However, they are all quite similar in terms of forecasting errors. Overall, all the tested models almost always demonstrate at least the same quality as the random walk model. However, when making a forecast for a horizon of one quarter, many models perform worse than the naïve forecast.

The second benchmark, the autoregression model, performs better than some regularisation methods if the full data set is used. That said, it is always worse than other models if the left-hand boundary of the training sample is established at 2000Q1.

Table 2. RMSFE (2000Q1 is used as the left-hand boundary of the training sample)

\begin{tabular}{lccccccccc} 
Model & $\boldsymbol{h}=\mathbf{1}$ & $\boldsymbol{h}=\mathbf{2}$ & $\boldsymbol{h = 3}$ & $\boldsymbol{h = 4}$ & $\boldsymbol{h = 5}$ & $\boldsymbol{h}=\mathbf{6}$ & $\boldsymbol{h}=\mathbf{7}$ & $\boldsymbol{h}=\mathbf{8}$ \\
\hline Random walk & 1 & 1 & 1 & 1 & 1 & 1 & 1 \\
\hline AR & 1.00 & 1.09 & 1.20 & 1.17 & 1.16 & 1.13 & 1.02 & 0.88 \\
\hline Adaptive LASSO & 1.00 & 0.64 & 0.97 & 0.84 & 0.89 & 0.89 & 0.63 & 0.63 \\
\hline Elastic Net & 0.78 & 0.62 & 0.96 & 0.76 & 0.81 & 0.82 & 0.67 & 0.59 \\
\hline LASSO & 0.91 & $\mathbf{0 . 5 8}$ & 0.99 & 0.74 & 0.88 & 0.83 & 0.66 & 0.57 \\
\hline Post-LASSO & 0.94 & 0.72 & 1.03 & 0.91 & 1.04 & 0.98 & 0.75 & 0.63 \\
\hline Ridge & 0.88 & 0.64 & 0.79 & 0.78 & 0.76 & 0.79 & 0.68 & 0.66 \\
\hline Spike and Slab & 0.85 & 0.64 & 0.80 & 0.80 & 0.91 & 0.83 & 0.66 & 0.60 \\
\hline Boosting $(\eta=0.1)$ & 0.97 & 0.64 & 0.70 & 0.60 & 0.68 & $\mathbf{0 . 5 9}$ & 0.60 & $\mathbf{0 . 4 4}$ \\
\hline Boosting $(\eta=0.2)$ & 0.90 & 0.63 & $\mathbf{0 . 6 5}$ & 0.58 & 0.69 & 0.63 & 0.63 & 0.57 \\
\hline Boosting $(\eta=0.3)$ & 1.10 & 0.69 & 0.66 & 0.66 & $\mathbf{0 . 6 3}$ & $\mathbf{0 . 5 9}$ & 0.62 & 0.52 \\
\hline Boosting $(\eta=0.4)$ & 1.08 & 0.66 & 0.72 & $\mathbf{0 . 5 6}$ & 0.67 & 0.66 & $\mathbf{0 . 5 6}$ & 0.50 \\
\hline Random forest $(N=100)$ & $\mathbf{0 . 7 5}$ & 0.62 & 0.73 & 0.62 & 0.67 & 0.63 & 0.62 & 0.56 \\
\hline Random forest $(N=500)$ & 0.81 & 0.61 & 0.73 & 0.64 & 0.67 & 0.63 & 0.61 & 0.58 \\
\hline Random forest $(N=1000)$ & 0.78 & 0.62 & 0.72 & 0.63 & 0.66 & 0.62 & 0.59 & 0.57 \\
\hline Random forest $(N=2000)$ & 0.78 & 0.62 & 0.73 & 0.64 & 0.66 & 0.62 & 0.59 & 0.58 \\
\hline
\end{tabular}

Tables 1 and 2, which demonstrate the quality of the models, may be amended using the Diebold-Mariano test described in Diebold and Mariano $(1995,2002)$ in order to compare the quality of the models. Since the size of test samples is quite small (17-25 observations), the statistics for the Diebold-Mariano test adjusted for small samples were used, as suggested by Harvey et al. (1997). The null hypothesis of the test implies that two models have the same quality of forecasts. An alternative hypothesis for each specification is single-sided, i.e. it implies that the model with the least RMSFE across the sample produces higher-quality forecasts.

Figures 3 and 4 in Appendix demonstrate the results of Diebold-Mariano tests for different models trained based on the same sample and having the same forecast horizon. The colour green in the figures means that the model corresponding to the line is better than that corresponding to the column. The colour yellow denotes 
superiority of the model corresponding to the column. The lighter the colour, the greater the $p$-value is according to Diebold-Mariano test statistics, and hence, the smaller the difference between the two forecasts. If the $p$-value is higher than 0.1 , the difference between the two models is statistically insignificant, which is indicated by the colour white in the figures. It is clear that the location of the yellow and green cells in the square is symmetrical in relation to the main diagonal.

It can be seen that as the forecasting horizon increases (with $h>4$ ), the forecasts produced by the models are more and more different from each other, and ensemble methods show superiority over regularisation methods more frequently. However, even with $h=8$, the forecasts produced by almost all models are virtually identical statistically. The only exception is the AR model, the quality of which is reduced dramatically when transitioning to a trimmed sample, and the random walk model, which falls behind the majority of models across most forecasting horizons.

Despite the fact that the quality of machine learning methods increases as the forecasting horizon increases compared to random walk methods, the ability to retrieve useful information from the data seems to worsen. When average horizons are used $(h=4, \ldots, 6)$, all ensemble methods are dramatically better than regularisation methods. Nonetheless, in the case of two-year forecasting, this advantage becomes statistically imperceptible. In fact, this means that the data contain insufficient information for the models to generate two-year forecasts that are significantly different from the merely average level.

Figures 3 and 4 in Appendix also show how transitioning to a trimmed sample influences various methods: high-quality models (boosting and random forest) become even better, while the quality of regularisation methods, which are on the whole worse, does not show the same improvement rate, or even deteriorates.

Figure 2 in Appendix shows out-of-sample forecasts on horizons up to one year ( $h \leq 4)$, from 2013Q1 to 2019Q1, for the trimmed data set. Each separate forecast line on the graph corresponds to a single model training session. The difference in the quality of the models in terms of RMSFE is also confirmed by the graph: it looks like boosting and random forest are better at reproducing the shifts in the forecasted series. The graph displays the forecasts produced by boosting and random forest models for a single specification only, because visually the forecasts of identical models turn out to be similar to each other in case of changes in the parameters. The graph does not display any random walk forecasts because they are trivial and are obtained by means of shifting observations to the right.

\subsubsection{Two data sets}

Tables 1 and 2 show that the data from the pre-crisis period almost never help to improve the forecasts. Why is that? This is due to the fact that the pre-crisis observations are very volatile, the variable values are non-typical for subsequent observations, and, as a result, the models trained based on them begin to produce lower-quality data forecasts for the previous periods. Figure 1 in Appendix 
demonstrates the values for all variables used in the research. We can see that data variability is reduced from the beginning of the century (it is expected that during the crisis periods of 2008-2009 and 2014-2015 the series become volatile again).

The Diebold-Mariano test may be used to perform a more rigorous check of this forecast improvement hypothesis.

Table 3 displays changes in RMSFE relative to the random walk model when transitioning to the trimmed sample, and the brackets enclose the values of Diebold-Mariano statistics for checking the hypothesis of the congruence between two models. The alternative hypothesis is single-sided, i.e. it implies that the model with the smallest RMSFE indicator demonstrates the highest quality. Machine learning models that were trained based on the trimmed data produce forecasts at least as good as similar models based on enhanced data for the oneyear horizon $(h \leq 4)$. The only exception is the boosting model, which produces significantly worse forecasts with $h=1$.

Table 3. Diebold-Mariano test: two samples

\begin{tabular}{|c|c|c|c|c|c|c|c|c|}
\hline Модель & $h=1$ & $h=2$ & $h=3$ & $h=4$ & $h=5$ & $h=6$ & $h=7$ & $h=8$ \\
\hline $\mathrm{AR}$ & $\begin{array}{c}-0.082 \\
(0.195)\end{array}$ & $\begin{array}{c}-0.243 \\
(0.081)\end{array}$ & $\begin{array}{c}-0.413^{*} \\
(0.018)\end{array}$ & $\begin{array}{l}-0.441^{* *} \\
(0.002)\end{array}$ & $\begin{array}{l}-0.477^{* *} \\
(0.002)\end{array}$ & $\begin{array}{c}-0.458^{* *} \\
(0.008)\end{array}$ & $\begin{array}{c}-0.337^{*} \\
(0.021)\end{array}$ & $\begin{array}{c}-0.271^{*} \\
(0.021)\end{array}$ \\
\hline Adaptive LASSO & $\begin{array}{c}-0.004 \\
(0.080)\end{array}$ & $\begin{array}{l}0.264^{*} \\
(0.025)\end{array}$ & $\begin{array}{c}-0.181 \\
(0.073)\end{array}$ & $\begin{array}{c}-0.002 \\
(0.186)\end{array}$ & $\begin{array}{r}-0.144^{*} \\
(0.015)\end{array}$ & $\begin{array}{c}-0.121 \\
(0.461)\end{array}$ & $\begin{array}{c}-0.013 \\
(0.490)\end{array}$ & $\begin{array}{l}-0.128 \\
(0.126)\end{array}$ \\
\hline Elastic Net & $\begin{array}{l}0.218 \\
(0.412)\end{array}$ & $\begin{array}{l}0.230^{*} \\
(0.046)\end{array}$ & $\begin{array}{l}-0.116 \\
(0.128)\end{array}$ & $\begin{array}{l}0.041 \\
(0.453)\end{array}$ & $\begin{array}{l}-0.050 \\
(0.421)\end{array}$ & $\begin{array}{l}-0.072 \\
(0.379)\end{array}$ & $\begin{array}{l}0.027 \\
(0.249)\end{array}$ & $\begin{array}{l}-0.037 \\
(0.250)\end{array}$ \\
\hline LASSO & $\begin{array}{l}0.104 \\
(0.472)\end{array}$ & $\begin{array}{l}0.309^{*} \\
(0.037)\end{array}$ & $\begin{array}{l}-0.153 \\
(0.113)\end{array}$ & $\begin{array}{l}0.074 \\
(0.343)\end{array}$ & $\begin{array}{l}-0.056 \\
(0.403)\end{array}$ & $\begin{array}{c}-0.102 \\
(0.421)\end{array}$ & $\begin{array}{l}0.055 \\
(0.130)\end{array}$ & $\begin{array}{l}-0.021 \\
(0.288)\end{array}$ \\
\hline Post-LASSO & $\begin{array}{l}0.105 \\
(0.298)\end{array}$ & $\begin{array}{l}0.229 \\
(0.054)\end{array}$ & $\begin{array}{l}-0.229 \\
(0.126)\end{array}$ & $\begin{array}{l}0.022 \\
(0.176)\end{array}$ & $\begin{array}{c}-0.243^{*} \\
(0.014)\end{array}$ & $\begin{array}{l}-0.201 \\
(0.325)\end{array}$ & $\begin{array}{c}-0.025 \\
(0.298)\end{array}$ & $\begin{array}{l}-0.095 \\
(0.128)\end{array}$ \\
\hline Ridge & $\begin{array}{l}0.098 \\
(0.365)\end{array}$ & $\begin{array}{l}0.240^{*} \\
(0.050)\end{array}$ & $\begin{array}{l}0.087 \\
(0.246)\end{array}$ & $\begin{array}{l}0.092 \\
(0.189)\end{array}$ & $\begin{array}{l}0.041 \\
(0.162)\end{array}$ & $\begin{array}{l}-0.005 \\
(0.249)\end{array}$ & $\begin{array}{l}0.025 \\
(0.484)\end{array}$ & $\begin{array}{l}-0.051 \\
(0.217)\end{array}$ \\
\hline Spike and Slab & $\begin{array}{l}0.142 \\
(0.479)\end{array}$ & $\begin{array}{l}0.207 . \\
(0.063)\end{array}$ & $\begin{array}{l}0.022 \\
(0.302)\end{array}$ & $\begin{array}{l}-0.013 \\
(0.418)\end{array}$ & $\begin{array}{c}-0.139^{*} \\
(0.045)\end{array}$ & $\begin{array}{c}-0.083 \\
(0.381)\end{array}$ & $\begin{array}{c}-0.024 \\
(0.292)\end{array}$ & $\begin{array}{l}-0.021 \\
(0.442)\end{array}$ \\
\hline Boosting $(\eta=0.1)$ & $\begin{array}{c}-0.058 \\
(0.109)\end{array}$ & $\begin{array}{l}0.100 . \\
(0.083)\end{array}$ & $\begin{array}{l}0.113 \\
(0.107)\end{array}$ & $\begin{array}{l}0.066 . \\
(0.069)\end{array}$ & $\begin{array}{c}-0.011 \\
(0.421)\end{array}$ & $\begin{array}{c}-0.054 \\
(0.083)\end{array}$ & $\begin{array}{c}-0.147^{* *} \\
(0.006)\end{array}$ & $\begin{array}{l}0.178^{*} \\
(0.034)\end{array}$ \\
\hline Boosting $(\eta=0.2)$ & $\begin{array}{l}-0.033 \\
(0.175)\end{array}$ & $\begin{array}{l}0.131 . \\
(0.062)\end{array}$ & $\begin{array}{l}0.139^{*} \\
(0.023)\end{array}$ & $\begin{array}{l}0.132 \\
(0.128)\end{array}$ & $\begin{array}{l}-0.011 \\
(0.493)\end{array}$ & $\begin{array}{c}-0.162^{*} \\
(0.050)\end{array}$ & $\begin{array}{c}-0.136^{* *} \\
(0.006)\end{array}$ & $\begin{array}{l}0.024 \\
(0.134)\end{array}$ \\
\hline Boosting $(\eta=0.3)$ & $\begin{array}{c}-0.136^{*} \\
(0.042)\end{array}$ & $\begin{array}{l}0.097 \\
(0.220)\end{array}$ & $\begin{array}{l}0.022 \\
(0.224)\end{array}$ & $\begin{array}{l}0.047 \\
(0.055)\end{array}$ & $\begin{array}{l}0.099 \\
(0.112)\end{array}$ & $\begin{array}{l}-0.064 \\
(0.117)\end{array}$ & $\begin{array}{l}-0.091 \\
(0.120)\end{array}$ & $\begin{array}{l}0.082 \\
(0.114)\end{array}$ \\
\hline $\operatorname{Boosting}(\eta=0.4)$ & $\begin{array}{l}-0.067 \\
(0.102)\end{array}$ & $\begin{array}{l}0.048 \\
(0.207)\end{array}$ & $\begin{array}{l}0.092 \\
(0.170)\end{array}$ & $\begin{array}{l}0.126 . \\
(0.089)\end{array}$ & $\begin{array}{c}-0.061 \\
(0.079)\end{array}$ & $\begin{array}{c}-0.118^{*} \\
(0.021)\end{array}$ & $\begin{array}{l}-0.081 \\
(0.325)\end{array}$ & $\begin{array}{l}0.091 \\
(0.185)\end{array}$ \\
\hline Random forest $(N=100)$ & $\begin{array}{l}0.156 \\
(0.269)\end{array}$ & $\begin{array}{l}0.096^{*} \\
(0.041)\end{array}$ & $\begin{array}{l}0.024 \\
(0.275)\end{array}$ & $\begin{array}{l}0.026 \\
(0.124)\end{array}$ & $\begin{array}{l}0.032 \\
(0.203)\end{array}$ & $\begin{array}{c}-0.071 \\
(0.269)\end{array}$ & $\begin{array}{l}-0.076 \\
(0.219)\end{array}$ & $\begin{array}{l}0.009 \\
(0.147)\end{array}$ \\
\hline Random forest $(N=500)$ & $\begin{array}{l}0.074 \\
(0.448)\end{array}$ & $\begin{array}{l}0.096^{*} \\
(0.036)\end{array}$ & $\begin{array}{l}0.026 \\
(0.353)\end{array}$ & $\begin{array}{l}0.019 . \\
(0.093)\end{array}$ & $\begin{array}{c}-0.011 \\
(0.392)\end{array}$ & $\begin{array}{c}-0.052 \\
(0.200)\end{array}$ & $\begin{array}{c}-0.071 \\
(0.079)\end{array}$ & $\begin{array}{l}-0.020 \\
(0.356)\end{array}$ \\
\hline Random forest $(N=1000)$ & $\begin{array}{l}0.114 \\
(0.308)\end{array}$ & $\begin{array}{l}0.075^{*} \\
(0.040)\end{array}$ & $\begin{array}{l}0.034 \\
(0.279)\end{array}$ & $\begin{array}{l}0.029 . \\
(0.093)\end{array}$ & $\begin{array}{l}-0.005 \\
(0.486)\end{array}$ & $\begin{array}{l}-0.044 \\
(0.224)\end{array}$ & $\begin{array}{l}-0.047 \\
(0.256)\end{array}$ & $\begin{array}{l}-0.019 \\
(0.369)\end{array}$ \\
\hline Random forest $(N=2000)$ & $\begin{array}{l}0.124 \\
(0.227)\end{array}$ & $\begin{array}{l}0.083^{*} \\
(0.037)\end{array}$ & $\begin{array}{l}0.027 \\
(0.337)\end{array}$ & $\begin{array}{l}0.027 \\
(0.101)\end{array}$ & $\begin{array}{l}0.008 \\
(0.385)\end{array}$ & $\begin{array}{c}-0.044 \\
(0.213)\end{array}$ & $\begin{array}{c}-0.040 \\
(0.199)\end{array}$ & $\begin{array}{l}-0.013 \\
(0.335)\end{array}$ \\
\hline
\end{tabular}

Note: the table shows change in RMSFE relative to the random walk model when switching to the trimmed sample. Inside the brackets is the $p$-value of Diebold-Mariano statistics $\left(H_{0}\right.$ : increasing the training sample by adding data from before the 1998 crisis does not affect the quality of the models). $p$-value: ${ }^{* *} \leq 0.001<^{* *} \leq 0.01<{ }^{*} \leq 0.05<. \leq 0.1$. 
With regard to two-year forecasts, the quality of several models also deteriorates with $h=5,6$, but neither of the two specifications is continuously superior to the other one. However, considering an increase in $h$, i.e. an increase in the gap between the dates on which and for which the forecast is made, the interpretability of predictions must decrease due to natural reasons, we can posit that removing the observations from before 1998 that are non-typical for subsequent periods makes it possible to produce more robust and adequate results when generating forecasts for at least one year ahead.

Out of the regularisation methods the Ridge model was the most robust with respect to sample trimming: either the deterioration in quality was insignificant $(h=6,8)$, or the quality actually improved. This was to be expected, considering that Ridge produces quite robust estimates.

However, the autoregression model shows significant deterioration with smaller sample. It seems that the effect of insufficient information in the course of sample trimming in this case exceeds the effect of adding atypical observations to the sample.

\subsubsection{Selection of variables in the LASSO model}

As we have already mentioned above, LASSO-based methods only allow the selection of the most important predictors. Despite that fact that LASSO did not perform well in the forecasting experiment for this research, the results of selecting the relevant predictor variables turned out to be interpretable and consistent with the basic theoretical idea of how the investment function may look. In this regard, it is important to briefly introduce the results of such selection.

Let us start with showing the number of variables that were selected by LASSO for various forecasting horizons and various training samples. Figure 5 in Appendix shows the number of LASSO-selected variables for the two different left-hand boundaries of the training sample.

The number of selected variables is quite unstable, which, according to Zou and Hastie (2005) and De Mol et al. (2008), is typical of LASSO models. In this case, we can see that for $h=3,5$, the number of selected variables is more robust for the models that were trained based on the data from 1996Q1 (based on the larger sample), and LASSO shows quality deterioration for these horizons if the sample is reduced (see Table 3). For $h=2$, on the contrary, the number of coefficients in the model that was trained based on a trimmed sample does not show as much fluctuation as the model trained based on the larger sample. This is consistent with the statistically significant improvement in model quality in the case of sample trimming.

However, what predictors are actually selected? Tables 4 and 5 show the values for the first five standardised values of the LASSO model coefficients specified in the module for various forecasting horizons with the start date of 2000Q1. The coefficients are standardised, so their values do not have a direct interpretation, but the greater the predictor's contribution to the changes in investment, the greater the absolute value of the coefficient. 
In the case of generating forecasts for a horizon of one quarter, the major predictors are the current GDP and the current lag of the explained variable, which is consistent with accelerator theory as described by Clark (1917) and Guitton (1955). According to this theory, there is an optimal ratio $\mu$ of capital to output, and companies aiming to maximise their profit strive to achieve this ratio. Adjustment occurs with lags, so the current level of investments must depend not only on the current output, but also on previous investments and previous output.

Key predictors for the remaining horisons $h$ can be found in Tables 4 and 5, however, forecast interpretability seems to decrease as the horizon increases. The most important variables for mid-term forecasting are CPI and accounts payable.

Table 4. Primary predictors in the LASSO model for $h=1, \ldots, 4$ (2000Q1 as the left-hand boundary of the training sample)

\begin{tabular}{|c|c|c|c|c|}
\hline & $h=1$ & $h=2$ & $h=3$ & $h=4$ \\
\hline 1 & $\begin{array}{l}\text { GDP in constant prices } \\
0.046\end{array}$ & $\begin{array}{l}\text { M2 (as of the end of the } \\
\text { quarter) } \\
0.023\end{array}$ & $\begin{array}{l}\text { Index of real retail trade } \\
\text { turnover } \\
0.228\end{array}$ & $\begin{array}{l}\text { Consumer price index } \\
\text { (CPI) } \\
-0.292\end{array}$ \\
\hline 2 & $\begin{array}{l}\text { Gross fixed capital } \\
\text { formation } \\
0.035\end{array}$ & $\begin{array}{l}\text { Household real money } \\
\text { income index } \\
0.023\end{array}$ & $\begin{array}{l}\text { Accounts payable } \\
\text { (average per quarter) } \\
-0.059\end{array}$ & $\begin{array}{l}\text { Accounts payable } \\
\text { (average per quarter) } \\
-0.083\end{array}$ \\
\hline 3 & $\begin{array}{l}\text { Household real money } \\
\text { income index } \\
0.024\end{array}$ & $\begin{array}{l}\text { Real salary index } \\
-0.015\end{array}$ & $\begin{array}{l}\text { M2 (as of the end of the } \\
\text { quarter) } \\
0.052\end{array}$ & $\begin{array}{l}\text { Real salary index } \\
-0.068\end{array}$ \\
\hline 4 & $\begin{array}{l}\text { M2 (as of the end } \\
\text { of the quarter) } \\
0.010\end{array}$ & $\begin{array}{l}\text { Construction price index } \\
0.014\end{array}$ & $\begin{array}{l}\text { Real salary index } \\
-0.042\end{array}$ & $\begin{array}{l}\text { Household real money } \\
\text { income index } \\
0.056\end{array}$ \\
\hline 5 & $\begin{array}{l}\text { Construction price index } \\
0.007\end{array}$ & $\begin{array}{l}\text { Import } \\
0.016\end{array}$ & $\begin{array}{l}\text { Share of gross fixed } \\
\text { capital formation in GDP } \\
\text { (nominal value) } \\
-0.036\end{array}$ & $\begin{array}{l}\text { Construction price index } \\
-0.051\end{array}$ \\
\hline
\end{tabular}

Table 5. Primary predictors in the LASSO model for $h=5, \ldots, 8$ (2000Q1 as the left-hand boundary of the training sample)

\begin{tabular}{|c|c|c|c|c|}
\hline & $h=1$ & $h=2$ & $h=3$ & $h=4$ \\
\hline 1 & $\begin{array}{l}\text { Consumer price index } \\
\text { (CPI) } \\
-0.022\end{array}$ & $\begin{array}{l}\text { Consumer price index } \\
\text { (CPI) } \\
-0.04\end{array}$ & $\begin{array}{l}\text { Consumer price index } \\
(\mathrm{CPI}) \\
-0.019\end{array}$ & $\begin{array}{l}\text { Accounts payable } \\
\text { (average per quarter) } \\
-0.013\end{array}$ \\
\hline 2 & $\begin{array}{l}\text { Accounts payable } \\
\text { (average per quarter) } \\
-0.007\end{array}$ & $\begin{array}{l}\text { Accounts payable } \\
\text { (average per quarter) } \\
-0.01\end{array}$ & $\begin{array}{l}\text { Accounts payable } \\
\text { (average per quarter) } \\
-0.011\end{array}$ & $\begin{array}{l}\text { Accounts receivable } \\
\text { (average per quarter) } \\
0.009\end{array}$ \\
\hline 3 & $\begin{array}{l}\text { Household real money } \\
\text { income index } \\
0.003\end{array}$ & $\begin{array}{l}\text { Construction price index } \\
-0.003\end{array}$ & $\begin{array}{l}\text { Construction price index } \\
-0.006\end{array}$ & $\begin{array}{l}\text { Consumer price index } \\
(\mathrm{CPI}) \\
0.007\end{array}$ \\
\hline 4 & $\begin{array}{l}\text { Construction price index } \\
-0.003\end{array}$ & $\begin{array}{l}\text { Accounts receivable } \\
\text { (average per quarter) } \\
0.003\end{array}$ & $\begin{array}{l}\text { Accounts receivable } \\
\text { (average per quarter) } \\
0.006\end{array}$ & $\begin{array}{l}\text { Index of real retail trade } \\
\text { turnover } \\
0.004\end{array}$ \\
\hline 5 & $\begin{array}{l}\text { Overdue accounts } \\
\text { receivable } \\
\text { (average per quarter) } \\
0.001\end{array}$ & $\begin{array}{l}\text { Industrial producer } \\
\text { prices index } \\
-0.002\end{array}$ & $\begin{array}{l}\text { Share of gross fixed } \\
\text { capital formation in GDP } \\
\text { (nominal value) } \\
-0.003\end{array}$ & $\begin{array}{l}\text { Industrial producer } \\
\text { prices index } \\
-0.004\end{array}$ \\
\hline
\end{tabular}

It is worth paying attention to the variable of the share of gross fixed capital formation in GDP, which is important for several horizons with a negative sign. 
The sign of this variable means that if in some quarter the growth of investments is higher than the growth of GDP so that the share of investments in GDP grow, then some quarters ahead a significantly negative effect on the growth of a forecasted variable is expected.

Despite the fact that the coefficients and their signs selected by LASSO do not contradict economic theory, the set of predictors is generally not stable. The same picture is observed for LASSO modifications. Probably, the latter explains why forecasts produced by them are inferior in quality as compared to the ensemble methods.

\subsection{Comparison with the MED forecast}

The MED publishes extensive forecasts of social and economic development indicators ${ }^{5}$ each autumn. The author compares the forecasting results produced by his own models with the forecasts of gross fixed capital formation growth rates released by the MED for the following year. The MED forecasts changes in percentage terms, so the author's forecasts were also re-calculated as percentage changes relative to the previous year. Thus, Q3 ${ }^{6}$ of year $t$ was used as the forecast date for year $t+1$, i.e. the forecast of annual change was calculated based on forecasts for $h=2$ (corresponding to Q1 of year $t+1$ ) etc., and $h=5$ (corresponding to $\mathrm{Q} 4$ of year $t+1$ ).

The earliest date from which out-of-sample annual forecasts are available is 2013Q3, and thus comparison with the MED forecast is possible starting from 2014.

Figure 6 in Appendix shows the forecasts issued by the MED and the author's models. Of all the specifications of ensemble methods, only one specification per method is displayed. The forecasts produced with different parameter values selected are visually similar to each other. We can see that, on the whole, neither of the two forecasting methods is an undisputed winner, however, boosting and random forest models are almost always close to the actual values of change in investments, which is consistent with their superior quality in terms of RMSFE.

\section{Conclusion}

In the research, forecasts for the gross fixed capital formation index in Russia were generated using a range of machine learning methods and an extensive set of predictors. The highest quality is demonstrated by ensemble methods (random forest and boosting), which outperform both simple reference models and regularisation methods. This is consistent with the literature on macroeconomic

\footnotetext{
${ }^{5}$ MED forecasts are available at https://economy.gov.ru/material/directions/makroec/

6 The forecast date (Q3) is established so as to ensure that the information used to train the forecasting models roughly corresponds to the information available as of the date of publication of the MED forecast (this usually takes place in November). For the purpose of clarity, the author assumes that the values of all predictors in Q3 are published no later than in Q4.
} 
forecasting. Using an enlarged data set that includes observations for the period before the 1998 crisis almost never allows the improvement of forecasts as compared with the trimmed data set. The relatively low quality of the forecasts produced by regularisation methods corresponds to the instability of the specifications they produce over time. According to the results of comparing forecasts obtained by the author and by the MED, some of the machine learning models yield much higherquality forecasts of short-term changes in investments.

A promising area for further research could be to extend the set of forecasting models and perform nowcasting of investments taking into account the nonsimultaneity in the release of different economic statistics data (the so-called 'ragged edge' issue).

Appendix is available at http://rjmf.econs.online/en; dx.doi.org/10.31477/rjmf.202001.35

\section{References}

Aganbegyan, A. (2016). Reduction of Investments - Death for the Economy, Investment Growth - its Salvation. Economicheskie Strategii, 18(4), pp. 74-83. [In Russian]. Available at: http://www.inesnet.ru/article/sokrashhenie-investicij-gibel-dlyaekonomiki-podem-investicij-ee-spasenie/ [accessed on 11 February 2010].

Bai, J. and Ng, S. (2008). Forecasting Economic Time Series Using Targeted Predictors. Journal of Econometrics, 146(2), pp. 304-317.

Baybuza, I. (2018). Inflation Forecasting Using Machine Learning Methods. Russian Journal of Money and Finance, 77(4), pp. 42-59. doi: 10.31477/rjmf.201804.42

Belloni, A. and Chernozhukov, V. (2011a). High Dimensional Sparse Econometric Models: An Introduction. In: P. Alquier, E. Gautier and G. Stoltz, eds., Inverse Problems and High-Dimensional Estimation. Springer Verlag Berlin Heidelberg, pp. 121-156.

Belloni, A. and Chernozhukov, V. (2011b). 11-penalized Quantile Regression in High-dimensional Sparse Models. Annals of Statistics, 39(1), pp. 82-130. doi: 10.1214/10-AOS827

Clark, J. M. (1917). Business Acceleration and the Law of Demand: A Technical Factor in Economic Cycles. Journal of Political Economy, 25(3), pp. 217-235.

De Mol, C., Giannone, D. and Reichlin, L. (2008). Forecasting Using a Large Number of Predictors: Is Bayesian Shrinkage a Valid Alternative to Principal Components? Journal of Econometrics, 146(2), pp. 318-328.

Diebold, F. X. and Mariano, R. S. (1995). Comparing Predictive Accuracy. Journal of Business and Economic Statistics, 13(3), pp. 253-263. doi: 10.1080/07350015.1995.10524599

Diebold, F. X. and Mariano, R. S. (2002). Comparing Predictive Accuracy. Journal of Business and Economic Statistics, 20(1), pp. 134-144. doi: 10.1198/073500102753410444 
Faust, J. and Wright, J. H. (2013). Forecasting Inflation. In: Elliott G. and Timmerman A., eds., Handbook of Economic Forecasting, Vol. 2. North-Holland: Elsevier, pp. 2-56.

Fokin, N. and Polbin, A. (2019). Forecasting Russia's Key Macroeconomic Indicators with the VAR-LASSO Model. Russian Journal of Money and Finance, 78(2), pp. 67-93. doi: 10.31477/rjmf.201902.67

Friedman, J. H. (2001). Greedy Function Approximation: A Gradient Boosting Machine. Annals of Statistics, 29(5), pp. 1189-1232.

Guitton, H. (1955). Koyck (L.M.) - Distributed Lags and Investment Analysis. Revue Économique, 6(1), pp. 127-128.

Harvey, D., Leybourne, S. and Newbold, P. (1997). Testing the Equality of Prediction Mean Squared Errors. International Journal of Forecasting, 13(2), pp. 281-291.

Hoerl, A. E. and Kennard, R. W. (1970). Ridge Regression: Biased Estimation For Nonorthogonal Problems. Technometrics, 12(1), pp. 55-67. doi: $10.1080 / 00401706.1970 .10488634$

Idrisov, G. and Sinelnikov-Murylev, S. (2014). Forming Sources of Long-run Growth: How to Understand Them? Voprosy Ekonomiki, 3, pp. 4-20. [In Russian]. doi: 10.32609/0042-8736-2014-3-4-20

Ishwaran, H. and Rao, J. S. (2005). Spike and Slab Variable Selection: Frequentist and Bayesian Strategies. Annals of Statistics, 33(2), pp. 730-773. doi:10.1214/009053604000001147

Kudrin, A. and Gurvich, E. (2014). A New Growth Model for the Russian Economy. Voprosy Ekonomiki, 12, pp. 4-36. [In Russian]. doi: 10.32609/0042-8736-2014-12-4-36

Kvisgaard, V. H. (2018) Predicting the Future Past. How Useful Is Machine Learning in Economic Short-Term Forecasting? Master thesis, University of Oslo.

Li, J. and Chen, W. (2014). Forecasting Macroeconomic Time Series: LASSO-Based Approaches and Their Forecast Combinations with Dynamic Factor Models. International Journal of Forecasting, 30(4), pp. 996-1015.

Liaw, A. and Wiener, M. (2002). Classification and Regression by randomForest. R News, 2(3), pp. 18-22.

Oreshkin, M. (2018). Prospects of Economic Policy. Ekonomicheskaya Politika, 2018, 13(3), pp. 8-27. [In Russian]. doi: 10.18288/1994-5124-2018-3-01

Santosa, F. and Symes, W. W. (1986). Linear Inversion of Band-Limited Reflection Seismograms. SIAM Journal on Scientific and Statistical Computing, 7(4), pp. 1307-1330. doi: 10.1137/0907087

Stock, J. H. and Watson, M. (2011). Dynamic Factor Models. In: M. Clements and D. Hendry, eds., The Oxford Handbook of Economic Forecasting. Oxford University Press.

Tibshirani, R. (1996). Regression Shrinkage and Selection Via the Lasso. Journal of the Royal Statistical Society: Series B (Methodological), 58(1), pp. 267-288. doi: 10.1111/j.2517-6161.1996.tb02080.x

Zou, H. (2006). The Adaptive Lasso and Its Oracle Properties. Journal of the American Statistical Association, 101 (476), pp. 1418-1429. doi: 10.1198/016214506000000735

Zou, H. and Hastie, T. (2005). Regularization and Variable Selection via the Elastic Net. Journal of the Royal Statistical Society: Series B (Statistical Methodology), 67(2), pp. 301-320. doi:10.1111/j.1467-9868.2005.00503. 